\title{
T-FLASH: TENSOR VISUALIZATION IN MEDICAL STUDIO
}

\author{
J. Wiklund ${ }^{1}$, V. Nicolas ${ }^{2}$, P. Rondao Alface ${ }^{2}$, M. Andersson ${ }^{1}$ and H. Knutsson $^{1}$ \\ ${ }^{1}$ Linköping University \\ Department of Biomedical Engineering \\ Medical Informatics \\ University Hospital SE-581 85 Linköping Sweden \\ \{jowi,matsa,knutte\}@imt.liu.se \\ ${ }^{2}$ Université catholique de Louvain \\ Communications and Remote Sensing Lab.
}

Place du Levant, 2, B-1348 Louvain-La-Neuve, Belgium

vincent.nicolas@uclouvain.be,patrice.rondao@uclouvain.be

\begin{abstract}
Tensor valued data are frequently used in medical imaging. For a 3-dimensional second order tensor such data imply at least six degrees of freedom for each voxel. The operators ability to perceive this information is of outmost importance and in many cases a limiting factor for the interpretation of the data. In this paper we propose a decomposition of such tensor fields using the Tflash tensor glyphs that intuitively conveys important tensor features to a human observer. A matlab implementation for visualization of single tensors are described in detail and a VTK/ITK implementation for visualization of tensor fields have been developed as a Medical Studio component.
\end{abstract}

\section{INTRODUCTION}

An efficient system development process is crucially dependent on the designers ability to interpret system behavior. In the present case the system output is a tensor field and the channel chosen to convey the information is the designers visual system. It is equally important that the information is presented in a way that is easily understood by the end user. In a medical setting it is paramount that the visualization techniques used are able to reduce the complicated tensor field information to relevant parameters that are useful in the clinical environment.

Tensor valued image data are frequently used to represent targeted structures in modern medical imaging analysis, e.g. diffusion tensors, strain tensors and local structure tensors. For second order tensors, the by far most common type today, the information stored in each voxel is a $3 \times 3$ tensor for 3-dimensional data. In the simple case of symmetric tensors this implies 6 degrees of freedom (inner dimensions). Vqisualizing such tensor fields is not a trivial task, showing volume slices of individual tensor components will, for example, not make much sense to a human. The tensor visualization problem is well recognized and a number of approaches have been presented, e.g. [3].

This paper presents new tensor visualization software, including a tensor glyph generator, that we believe to be preferable in many situations.

\section{THE TFLASH TENSOR GLYPH}

The tensor visualization tool presented in this paper is designed for visualization of 2:nd order symmetric tensor fields. Normally the tensor fields are dense. The standard way of decomposing a symmetric second order tensor is

$$
\boldsymbol{T}=\lambda_{1} \boldsymbol{e}_{1} \boldsymbol{e}_{1}^{T}+\lambda_{2} \boldsymbol{e}_{2} \boldsymbol{e}_{2}^{T}+\lambda_{3} \boldsymbol{e}_{3} \boldsymbol{e}_{3}^{T} \quad \lambda_{1} \geq \lambda_{2} \geq \lambda_{3}
$$



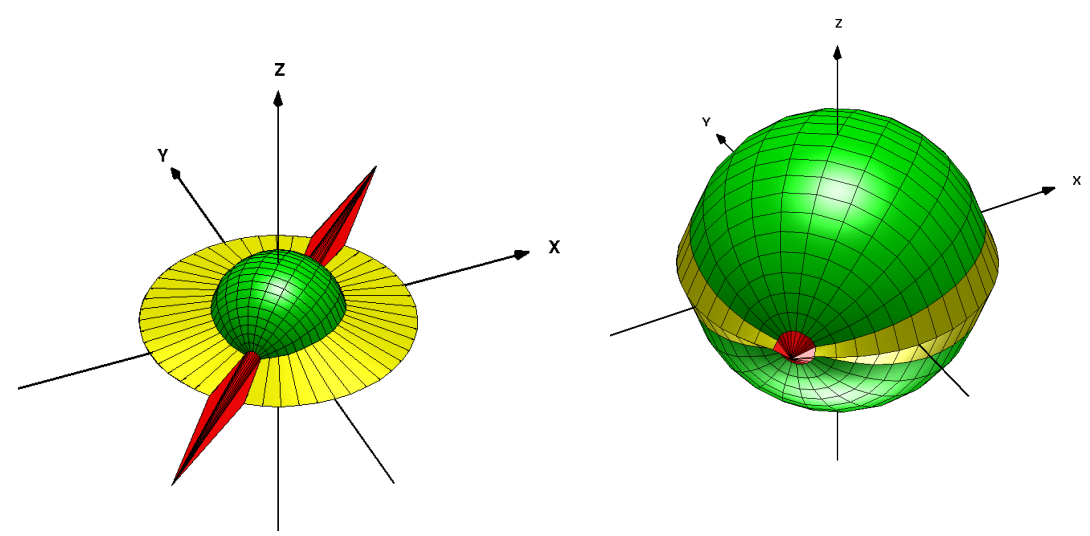

Fig. 1. Left: Tflash tensor glyph for $\lambda_{1}=1.00 \lambda_{2}=0.50 \lambda_{3}=0.25$, see text for details. Right: an almost isotropic tensor, $\lambda_{1}=0.67 \lambda_{2}=0.63 \lambda_{3}=0.59$

where $\lambda_{i}$ are the eigenvalues and $e_{i}$ the corresponding eigenvectors.

The glyph is inspired by the traditional decomposition of the (symmetric second order) local structure tensor into three parts, the linear part, $\boldsymbol{T}_{1}$, the planar part, $\boldsymbol{T}_{2}$, and the isotropic part, $\boldsymbol{T}_{3}$ where

$$
\boldsymbol{T}=\boldsymbol{T}_{1}+\boldsymbol{T}_{2}+\boldsymbol{T}_{3}
$$

where

$$
\begin{aligned}
& \boldsymbol{T}_{1}=\left(\lambda_{1}-\lambda_{2}\right) \boldsymbol{e}_{1} \boldsymbol{e}_{1}^{T} \\
& \boldsymbol{T}_{2}=\left(\lambda_{2}-\lambda_{3}\right)\left(\boldsymbol{e}_{1} \boldsymbol{e}_{1}^{T}+\boldsymbol{e}_{2} \boldsymbol{e}_{2}^{T}\right) \\
& \boldsymbol{T}_{3}=\lambda_{3}\left(\boldsymbol{e}_{1} \boldsymbol{e}_{1}^{T}+\boldsymbol{e}_{2} \boldsymbol{e}_{2}^{T}+\boldsymbol{e}_{3} \boldsymbol{e}_{3}^{T}\right)
\end{aligned}
$$

The tensor glyph is based on a parametric sphere surface rendering. The magnitude of the isotropic part corresponds to the radius of the sphere. The sphere is rendered in green, see fig. 1. The poles of the sphere are oriented in the direction of $\boldsymbol{e}_{1}$ and the planar part is visualized as a red spear through the poles where the length of the spear is proportional to the magnitude of $\boldsymbol{T}_{1}$. The red part of the glyph is generated by expanding the segments of the sphere located around the poles. Finally $\boldsymbol{T}_{2}$ is visualized by an expansion of the two longitudes of the sphere that corresponds to a plane orthogonal $\boldsymbol{e}_{3}$. This area of the glyph is rendered in yellow, see fig. 1 .

Since the Tflash glyph is based on ad distorted sphere the visualization becomes very efficient and the distinct shape together with the coloring improves the observers ability to perceive the geometry of the glyph from all views. The difficulties in perceiving the correct geometry using more fundamental glyphs as e.g. an ellipsoid is one of the main reasons for the introduction of the Tflash glyph.

To the right in fig. 1 another example of the Tflash tensor glyph is shown. The eigenvectors are identical to the left tensor but the distribution of the eigenvalues correspond to a much more isotropic tensor with $\lambda_{1}=0.67 \lambda_{2}=0.63 \lambda_{3}=0.59$. For the left tensor in fig. 2 the planar part, $\boldsymbol{T}_{2}$, is dominant $\left(\lambda_{1}=0.95 \lambda_{2}=0.90 \lambda_{3}=0.85\right)$. The yellow part is oriented in a direction orthogonal to $e_{3}$.

As the local structure tensor describes the directed energy distribution the Fourier domain there exists no physical interpretation of an indefinite local tensor. Negative eigenvalues $\left(\lambda_{3}<0\right)$ may, however, occur due to e.g. phase interference within the filters in complex or low SNR signal 

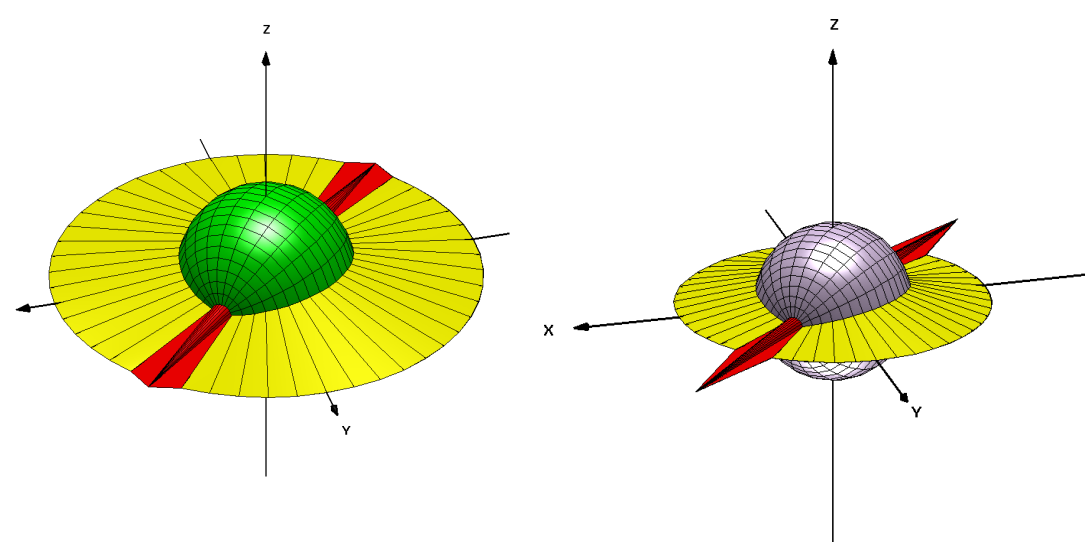

Fig. 2. Left: Planar part, $\boldsymbol{T}_{2}$, is dominant, $\lambda_{1}=0.90 \quad \lambda_{2}=0.85 \lambda_{3}=0.35$. Right: a negative $\lambda_{3}$ is visualized by a change in color of the isotropic part, $\lambda_{1}=1 \quad \lambda_{2}=0.6 \quad \lambda_{3}=-0.30$

neighborhoods. Although this is a relatively rare event it indicates that the second order tensor model is not sufficient to represent such a neighborhood and it is important to convey this information to the observer of the tensor glyph.

The shape of the Tflash tensor glyph is based on the absolute value of the eigenvalues but a negative sign of the eigenvalues cause a distinct change in the color rendering. In the right part of fig. $2 \lambda_{1}=1 \lambda_{2}=0.6 \lambda_{3}=-0.30$ which introduce a blue haze in the isotropic part. It would be straight forward to let the sign of the eigenvalues affect the shape of the glyph as well. This would on the other hand make the glyph more difficult to interpret and could cause ambiguities in certain views.

\section{IMPLEMENTATION AS A MEDICALSTUDIO COMPONENT}

MedicalStudio is a crossplatform framework for visualization, interaction and processing of medical images. It is based on popular open source libraries such as VTK for visualization, ITK for image processing, GTKmm for graphical user interface and DCMTK for Dicom compatibility. Intended to be flexible, the implementation allows an easy integration of new components. Mono and multimodal registration, atlas-based segmentation, 3D reconstruction, augmented visualization are some examples of the already available components.

Tensor field visualization by Tflash glyphs has been implemented on the basis of the vtkTensorGlyph class of VTK. Starting from a parameterized sphere primitive, the spear and circle are created by displacing the poles and a meridian in the normal direction according to the eigenvalues (see Fig. 3 ). The color code is implemented by adding face color attributes. The final orientation of the glyph is then obtained through a transform matrix defined by the tensor eigenvectors.

The integration into MedicalStudio is done by creating only one graphical component. This component drives the new TflashGlyphFilter and exposes its parameters to the user, which allows to easily test several configurations according to the loaded data. Visualization of the generated glyphs, navigation through the volume, data management, interaction management, ... a lot of components are already implemented into MedicalStudio, letting the programmer to concentrate on its main task.

\section{RESULT}

Figure 4 represents the Tflash glyph on a phantom regular grid with various eigenvalues sets and slowly varying orientations. This figure shows the capability of the Tflash to naturally highlight 
Similar NoE Tensor Workshop, Las Palmas, November 2006

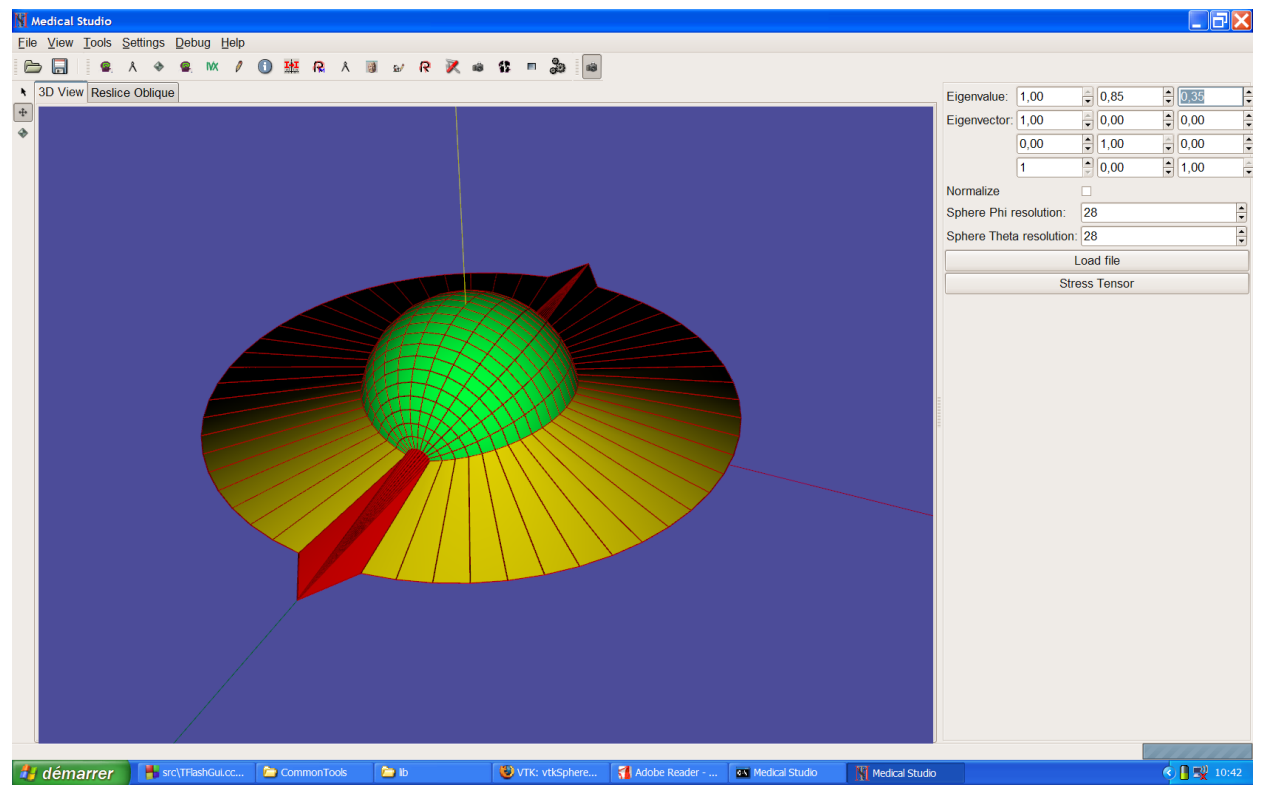

Fig. 3. A single glyph represented in MedicalStudio

global principal directions as well as to illustrate the various local properties of the tensor data. An illustration of the Tflash glyph on DTI tensor data is represented on Fig. 5.

When compared to other glyphs, the Tflash necessitates a very small amount of polygons to be rendered (i.e. as many as the well-known minimalist ellipsoids) while describing as much information as the superquadrics glyph [2]. This point facilitates a fast and fluid rendering of the tensor data. However, there is still no objective way to compare tensor visualization tools. Actually, benchmarking tools are strongly needed to test the performances of the state of the art tensor visualization techniques. Their performances mainly relate to the psycho-visual efficicacy as well as to the rendering efficiency. This is an open issue that deserves a common effort of the tensor processing community.

\section{CONCLUSION}

This paper has presented the Tflash glyph as an efficient tool to visualize tensor data. This glyph enables to univocally represent tensor eigenvector directions as well as the relative amplitudes of tensor eigenvalues in a very simple 3D model. The generation of the glyph is very simple and fast since it only necessitates the morphing of a very small number of points of a generic sphere. The color code is also simple and clear. This paper has also presented the MedicalStudio platform as well as the easy integration of the Tflash as a component. 
Similar NoE Tensor Workshop, Las Palmas, November 2006

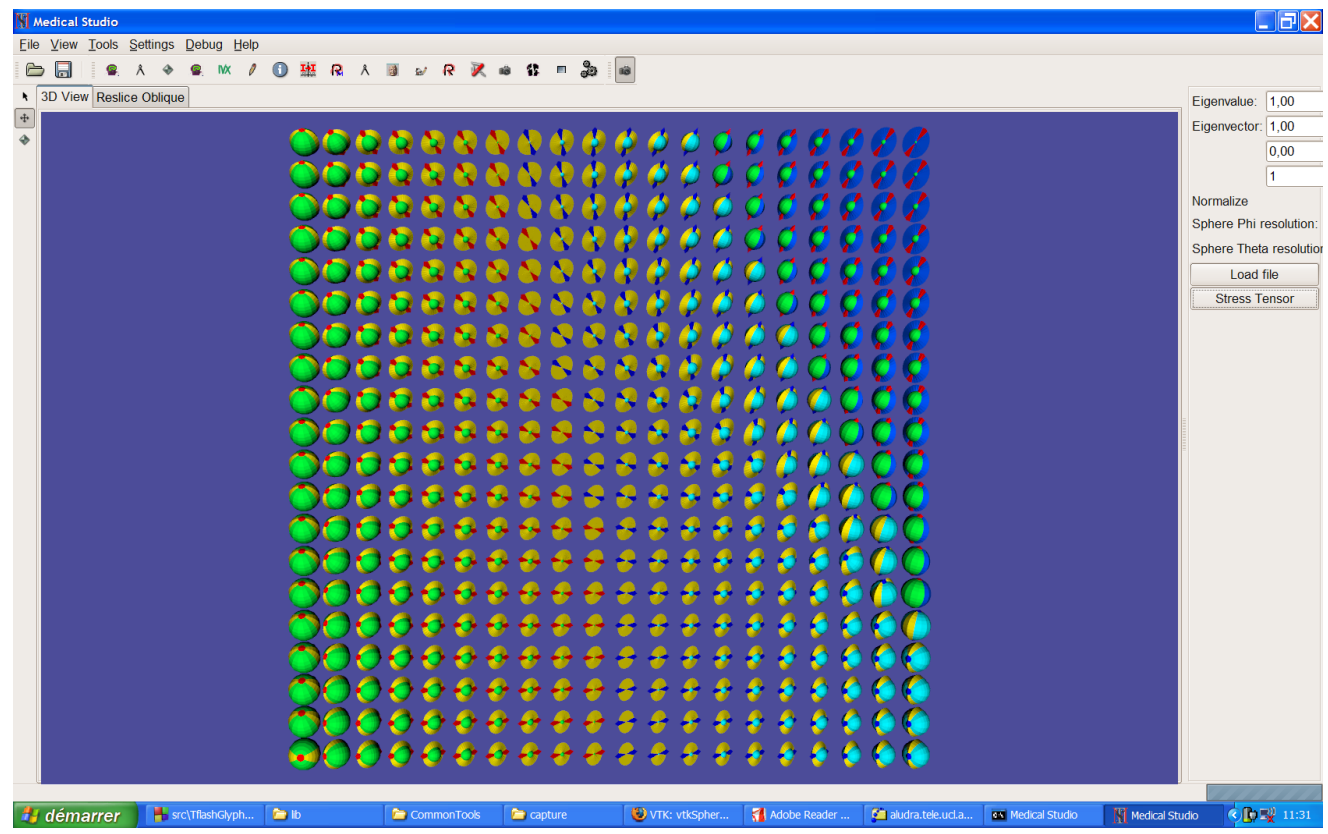

Fig. 4. Phantom regular grid illustrating some of the many possible configurations of a tensor glyph

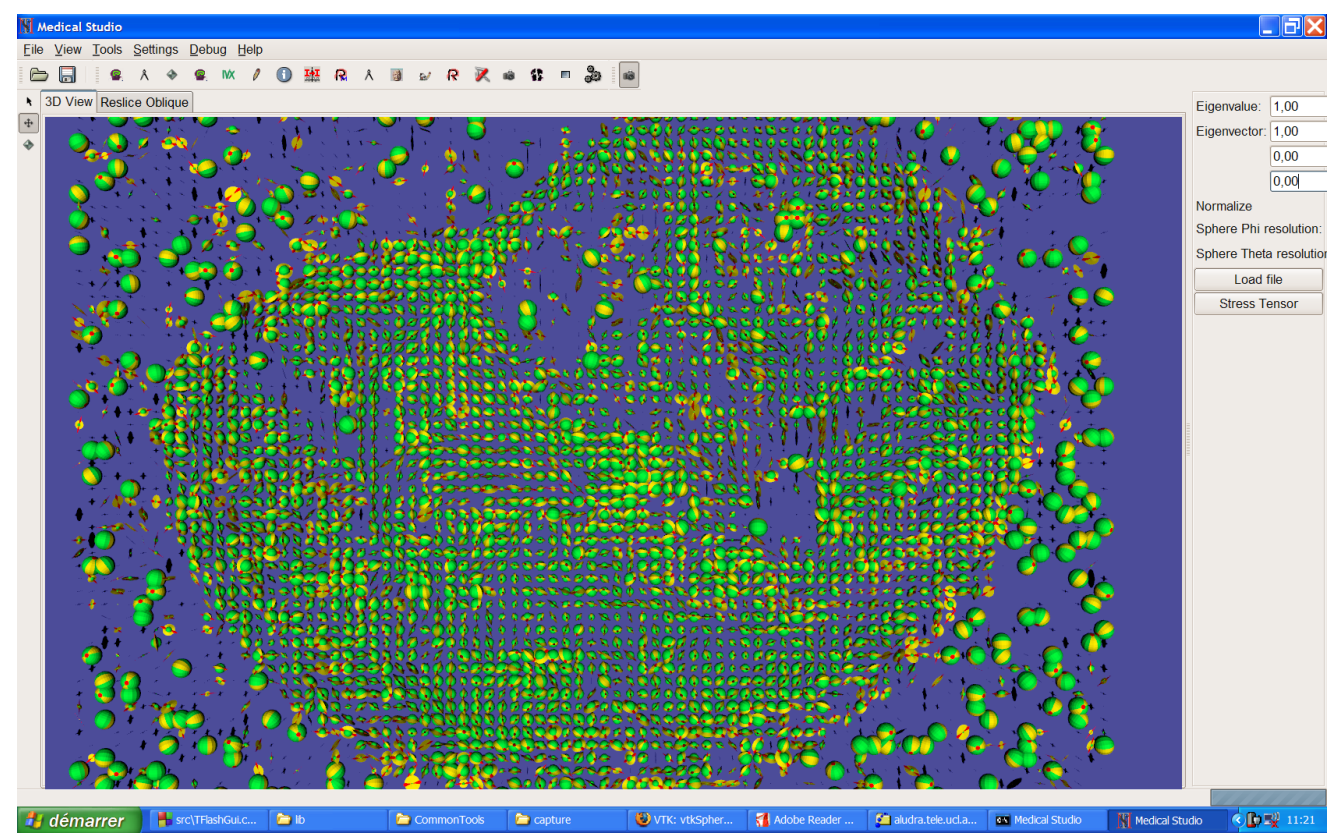

Fig. 5. Tflash and DTI tensor visualization in MedicalStudio 
Similar NoE Tensor Workshop, Las Palmas, November 2006

\section{A. MATLAB CODE FOR TFLASH}

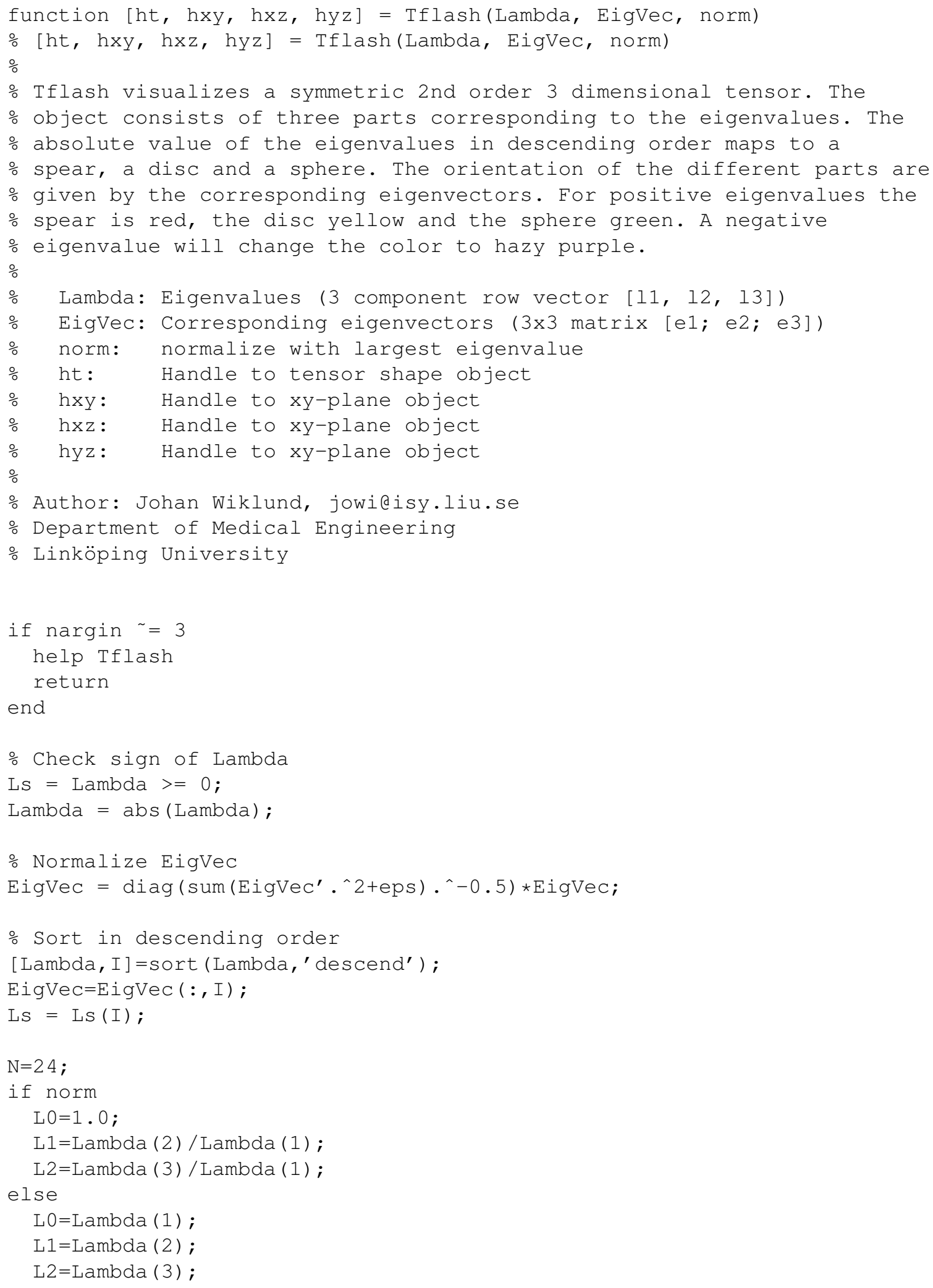




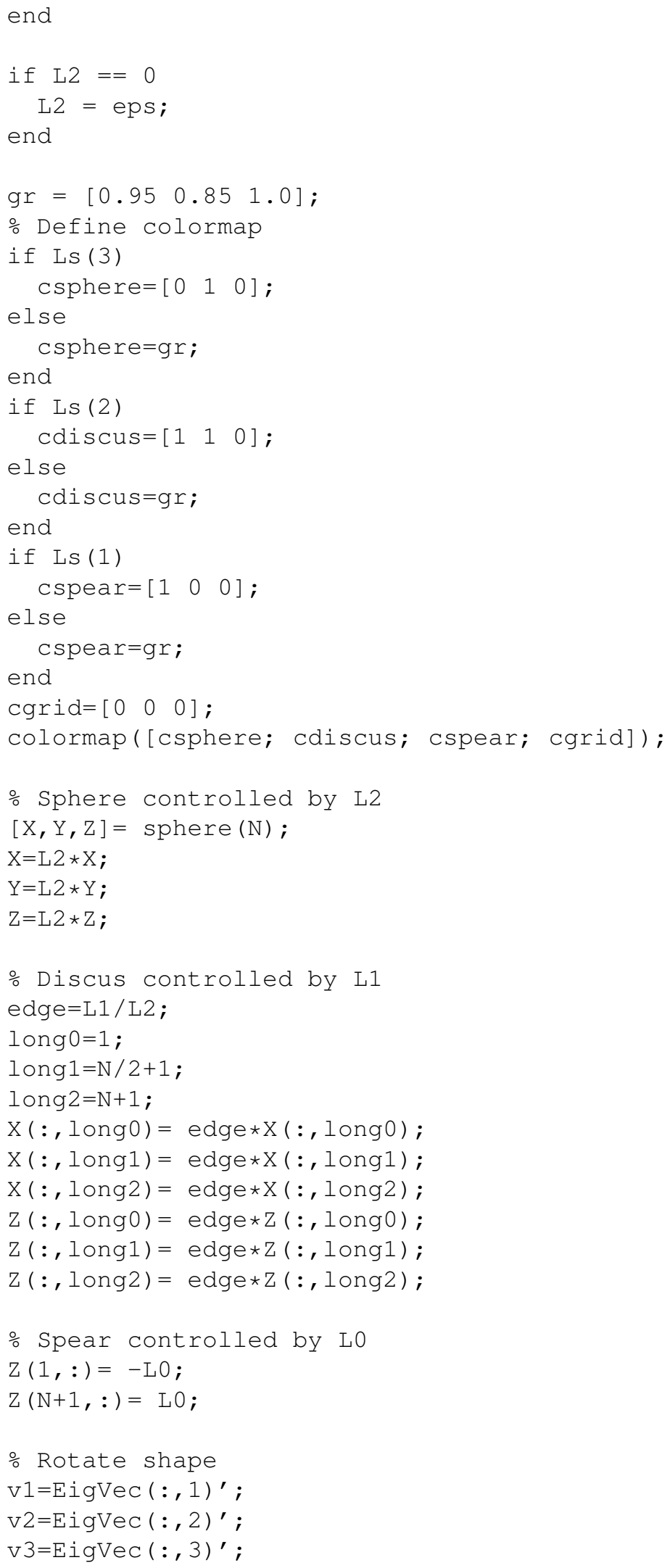




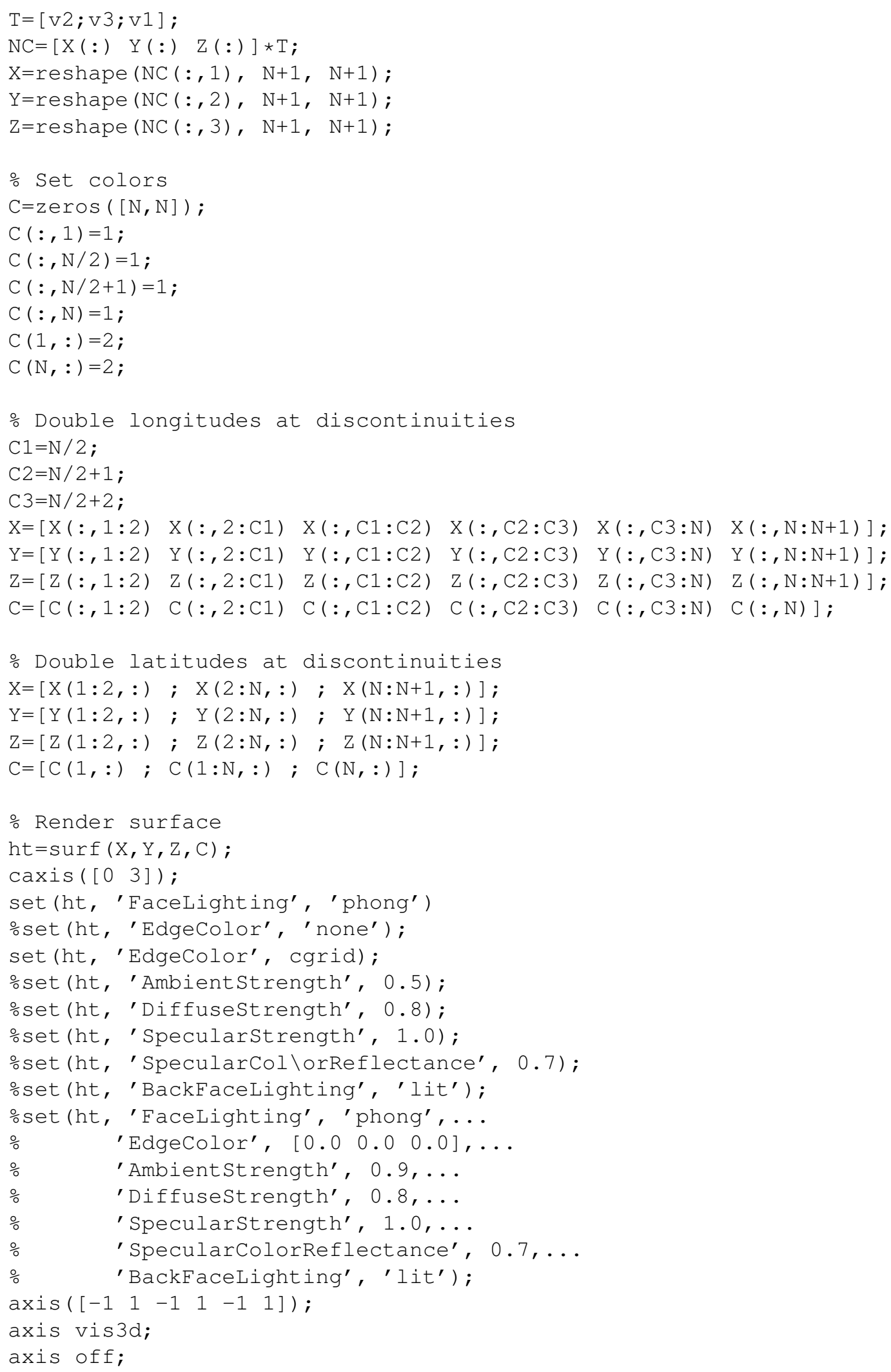




\section{Similar NoE Tensor Workshop, Las Palmas, November 2006}

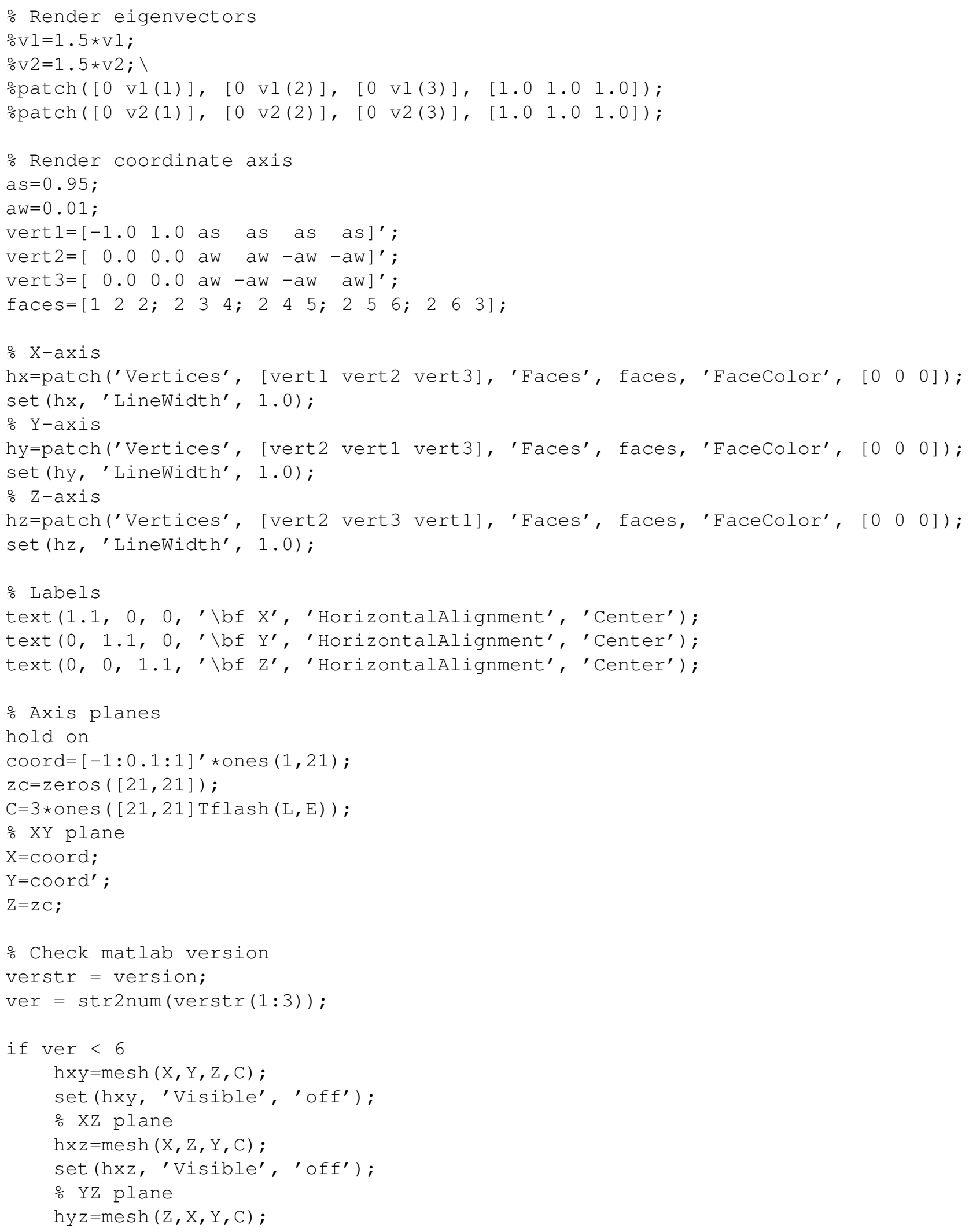




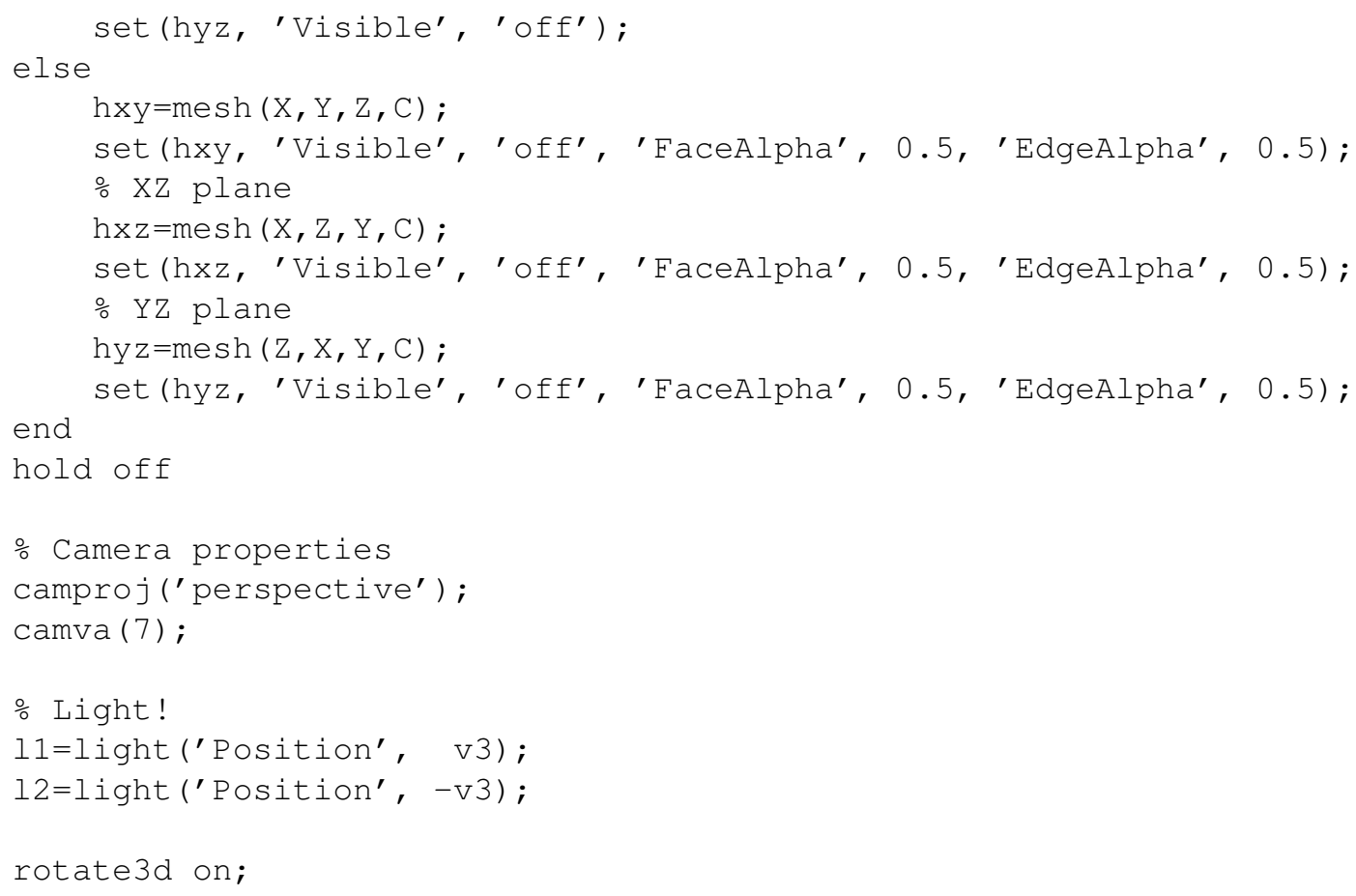

\section{B. REFERENCES}

[1] G. H. Granlund and H. Knutsson. Signal Processing for Computer Vision. Kluwer Academic Publishers, 1995. ISBN 0-7923-9530-1.

[2] Gordon Kindlmann. Superquadric tensor glyphs. In Proceeding of The Joint Eurographics IEEE TCVG Symposium on Visualization, pages 147-154, May 2004.

[3] Gordon Kindlmann. Visualization and Analysis of Diffusion Tensor Fields. PhD thesis, University of Utah, 2004.

[4] H. Knutsson. Representing local structure using tensors. In The 6th Scandinavian Conference on Image Analysis, pages 244-251, Oulu, Finland, June 1989. Report LiTH-ISY-I-1019, Computer Vision Laboratory, Linköping University, Sweden, 1989.

[5] V. Nicolas and B. Macq. Medical Studio. http://www.medicalstudio.org. 